\title{
A Novel Two-Color Photodetector Based on an InAlAs-InGaAs HEMT Layer Structure
}

\author{
M. Marso, M. Wolter, and P. Kordoš
}

\begin{abstract}
The spectral responsivity of an InAlAs-InGaAs metal-semiconductor-metal diode above a two-dimensional electron gas (2DEG) is investigated as a function of the applied bias. At low voltages, only the InAlAs layer above the 2DEG contributes to the photocurrent, while the InGaAs channel layer is activated at higher bias. This results in a voltage-dependent spectral response of the photodetector. The ratio of the responsivities at 1300 and $850 \mathrm{~nm}$ changes from $0.03-$ at $1-\mathrm{V}$ to $0.44-$ at $1.6-\mathrm{V}$ bias. This property makes the device a candidate suitable to detect and to separate optical information originated both from the GaAs $(850 \mathrm{~nm})$ and in the InGaAs $(1300,1550 \mathrm{~nm})$-based optoelectronic technology.
\end{abstract}

Index Terms-Infrared detectors, metal-semiconductor-metal (MSM) devices, MODFETs, photodetectors, spectroscopy.

\section{INTRODUCTION}

C OLOR IMAGE processing is usually performed with color-filter-coated photodetectors [1]. To overcome the splitting of one color pixel into several chromatic subpixels, different photodetector designs to detect the color information in one single device have been proposed. One concept uses a stack of p-n or p-i-n photodiodes, where the spectral sensitivity of the individual junctions is realized by the wavelengthdependent absorption coefficient of $\alpha$-Si: $\mathrm{H}$ alloys [2] or by the different penetration depths of red, green, and blue light in crystalline silicon [3]. By another approach, the spectral sensitivity of two-terminal devices depends on the applied bias voltage, as was demonstrated with GaAs-based multiquantum well infrared photodetectors [4]. In this work, we investigate the voltage-dependence of the spectral responsivity of a metalsemiconductor-metal diode above a two-dimensional electron gas (MSM-2DEG).

\section{DEVICE CONCEPT}

The MSM-2DEG has been investigated extensively for use as varactor diode and as photodetector [5]-[8]. It consists of two Schottky contacts above a high-electron mobility transistor (HEMT) layer system, simplifying integration in HEMT circuits (Fig. 1) [9]. The 2DEG with its high sheet carrier concentration (typically in the order of $10^{12} \mathrm{~cm}^{-2}$ for InAlAs-InGaAs based structures) acts as an equipotential plane that forces the electric field and the depletion layer of the reverse biased Schottky contact in the region between surface and 2DEG, provided that the

Manuscript received May 7, 2004; revised June 25, 2004.

The authors are with the Institute of Thin Films and Interfaces and CNI, Center of Nanoelectronic Systems for Information Technology, Research Centre Jülich, 52425 Jülich, Germany (e-mail: m.marso@fz-juelich.de).

Digital Object Identifier 10.1109/LPT.2004.834909

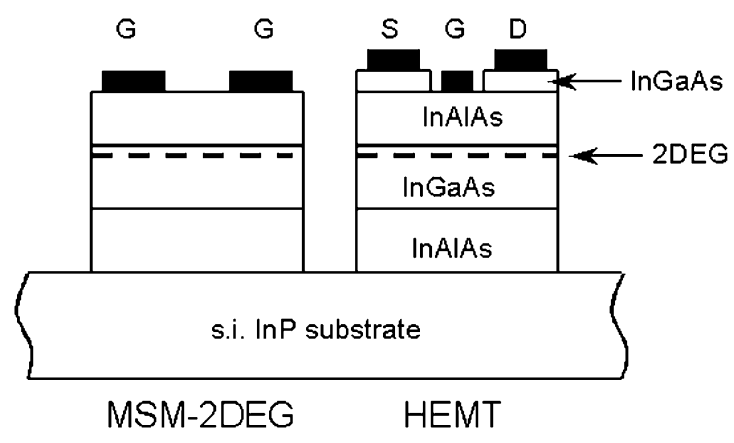

Fig. 1. Layer system and schematic diagram of an MSM-2DEG diode integrated with an HEMT.

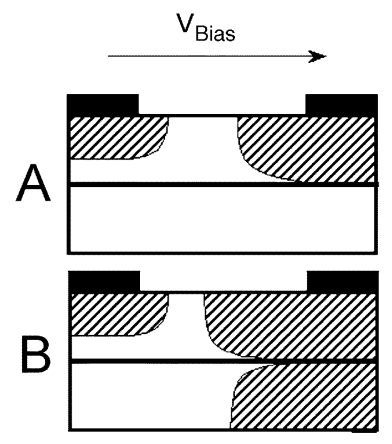

Fig. 2. Extension of the depletion zone for (A) low and (B) high bias voltage. The electrode on the right is reverse-biased.

bias voltage is not too high [Fig. 2(A)]. When the applied bias becomes sufficiently high to totally deplete the 2DEG channel below the contact then the depletion region penetrates the region below the channel [Fig. 2(B)]. This causes a dramatic decrease of the device capacitance that can be used for varactor applications [5]. When the device is used as photodetector, then only the carriers generated in the depletion region contribute to the photocurrent. In former publications, only the high-voltage regime was used for photodetector applications. An InAlAsInGaAs-based MSM-2DEG photodetector was published with a bandwidth of $20 \mathrm{GHz}$ by excitation with $1.3-\mu \mathrm{m}$ wavelength light [9]. For use as a two-color detector, we take advantage of the voltage-dependent penetration depth of the depletion region to control the spectral dependence of the photodetector responsivity by the applied voltage. In contrast to a conventional MSM without 2DEG, the penetration of the depletion region into the InGaAs layer is very abrupt and can be controlled by the carrier concentration in the channel. 


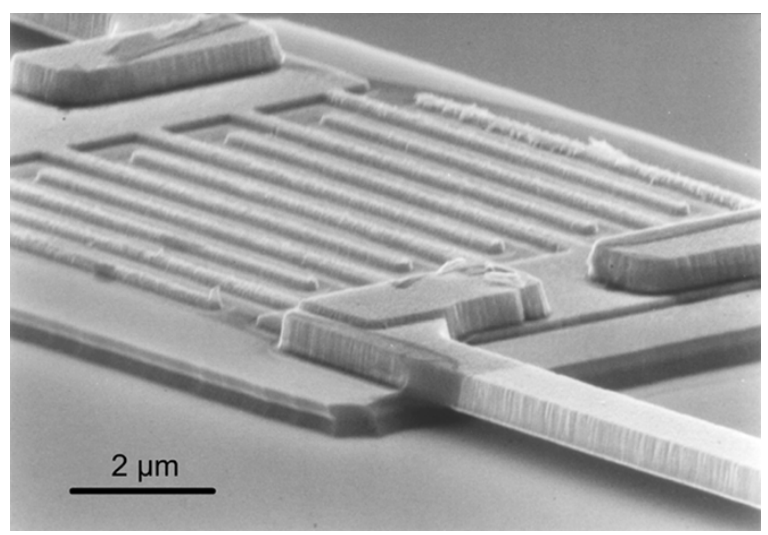

Fig. 3. Scanning electron microscope picture of an MSM-2DEG photodetector with airbridges to contact the electrodes. Device area is $10 \times 10 \mu \mathrm{m}^{2}$.

\section{DEVICE FABRICATION}

The HEMT layer sequence is grown by molecular beam epitaxy on a semi-insulating InP substrate. It consists of a 200-nm-thick InAlAs buffer layer, a 100-nm-thick InGaAs absorption layer, an 8-nm-thick strained $\operatorname{In}_{0.75} \mathrm{Ga}_{0.25}$ As channel layer, a 5-nm-thick InAlAs spacer, $3 \times 10^{12} \mathrm{~cm}^{-2} \mathrm{Si}$ delta doping, a 40-nm-thick InAlAs barrier layer, and a 10-nm-thick $\mathrm{n}^{+}$-doped InGaAs cap layer. All layers except the channel are lattice-matched to InP. Room-temperature Hall measurements yield a 2DEG carrier concentration of $1.8 \times 10^{12} \mathrm{~cm}^{-2}$ and a mobility of $7400 \mathrm{~cm}^{2} / \mathrm{Vs}$. The devices are prepared within the standard HEMT fabrication process (for details see [10]). The Schottky contacts are defined by e-beam lithography. They consist of an opaque $\mathrm{Ti}-\mathrm{Au}$ layer system with finger width and spacing of 300 and $600 \mathrm{~nm}$, respectively. The InGaAs cap layer is removed before metallization by succinic acid. Mesa etching is performed as last step after contact pad formation. The contact metallization at the edges of the mesa is only $1.5 \mu \mathrm{m}$ wide to obtain underetching during mesa formation. This technique avoids a shortcut of the contacts to the InGaAs absorption layer at the mesa edges (Fig. 3).

\section{Measurement Results}

All measurements are performed on a photodetector with $50 \times 50 \mu \mathrm{m}^{2}$ active area. The device is at first characterized by electrical dc and capacitance-voltage measurements with an AGILENT 4155B semiconductor parameter analyzer and an AGILENT 4294A impedance analyzer. The abrupt decrease of the capacitance at $1.1 \mathrm{~V}$ identifies the transition voltage where the 2DEG below the reverse biased electrode is completely emptied and where the depletion layer starts to penetrate the InGaAs absorption layer. The dark current is $1 \mu \mathrm{A}$ at $3-\mathrm{V}$ bias voltage. For optoelectronic measurements, a setup is used where the light from a Xenon arc lamp is directed through a grating monochromator. The monochromatic light is focused by a lens to the device under test. The measurement accuracy is improved by a lock-in amplifier including a chopper in the light beam. The power of the incident light is in the range of $1-3 \mu \mathrm{W}$, depending on wavelength. Because the active device area is smaller than the focused light spot, the measured responsivity is calibrated at $850-$ and $1310-\mathrm{nm}$ wavelength with

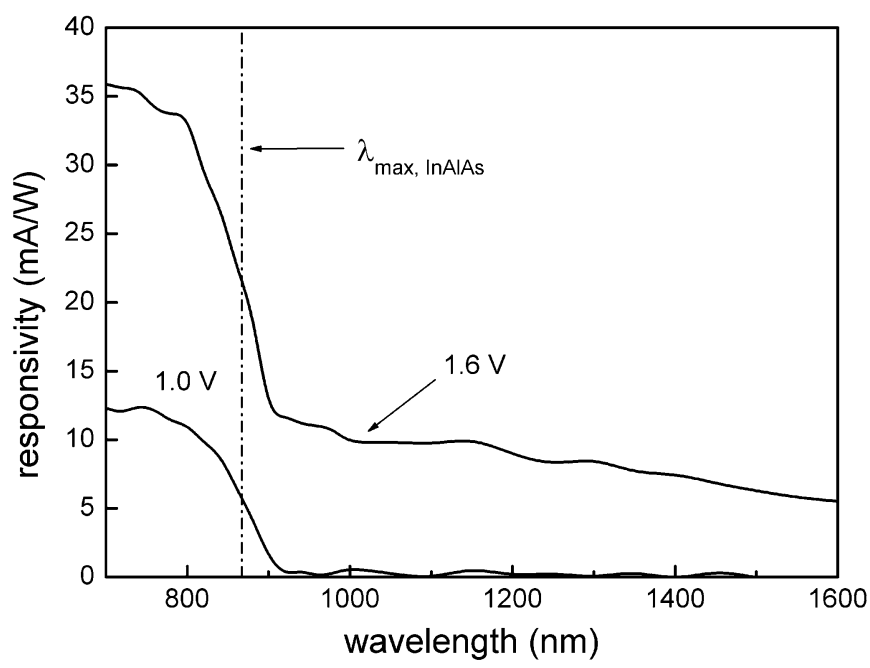

Fig. 4. Spectral responsivity for an applied bias below (1 V) and above (1.6 V) the transition voltage $(1.1 \mathrm{~V})$.

an HP 8153A lighwave multimeter, using a glass fiber with $9-\mu \mathrm{m}$ core diameter. The light that misses the active device area affects the measurement to less than $1 \%$ in the whole wavelength range, as determined by measurement of reference samples with similar contact pads as the photodetector, but without mesa or Schottky contacts. Fig. 4 shows the spectral responsivity for two different bias voltages. At $1 \mathrm{~V}$, the depletion region is stopped at the DEG channel. The electric field is limited to the InAlAs barrier layer with a bandgap of $1.43 \mathrm{eV}$ [11] that corresponds to a wavelength of $867 \mathrm{~nm}$. The responsivity drops from $12 \mathrm{~mA} / \mathrm{W}$ below $800 \mathrm{~nm}$ to less than $0.2 \mathrm{~mA} / \mathrm{W}$ above $900-\mathrm{nm}$ wavelength because the photocurrent is mainly created in the depletion region. The increase of the bias voltage to $1.6 \mathrm{~V}$ (above the transition voltage of $1.1 \mathrm{~V}$ ) extends the depletion layer into the InGaAs absorption layer. The responsivity is extended to the wavelength range up to $1700 \mathrm{~nm}$ that is the limit for lattice-matched InGaAs with a bandgap of $0.73 \mathrm{eV}$. This result demonstrates the property of the InAlAs-InGaAs-based MSM-2DEG photodetector that the responsivity can be switched ON and OFF in the wavelength range between 900 and $1700 \mathrm{~nm}$ by changing the bias voltage, while the responsivity below $900 \mathrm{~nm}$ only changes its value. In general, the device provides two linear independent spectral responses by measuring at two different bias voltages (one below and one above the transition voltage). This allows the separation of the incident light in two different colors, e.g., the determination of the intensities of two incident light beams with 850- and 1300-nm wavelength, respectively.

Fig. 5 depicts the responsivity as a function of the bias voltage for two selected wavelengths. The 850-nm wavelength light that excites both InAlAs and InGaAs creates a photocurrent already at very low bias voltages, while the 1300 -nm light activates the photodetector not until $1.1 \mathrm{~V}$, when the depletion region starts to penetrate the InGaAs absorption layer. The ratio $R_{1300} / R_{850}$ of the responsivities at 1300 and $850 \mathrm{~nm}$ is below 0.03 at low voltages. It begins to increase above $1.1 \mathrm{~V}$ and reaches a maximum value of 0.44 at $1.6 \mathrm{~V}$. For larger bias voltages, the responsivity at $850 \mathrm{~nm}$ increases faster than the value for $1300 \mathrm{~nm}$, resulting 


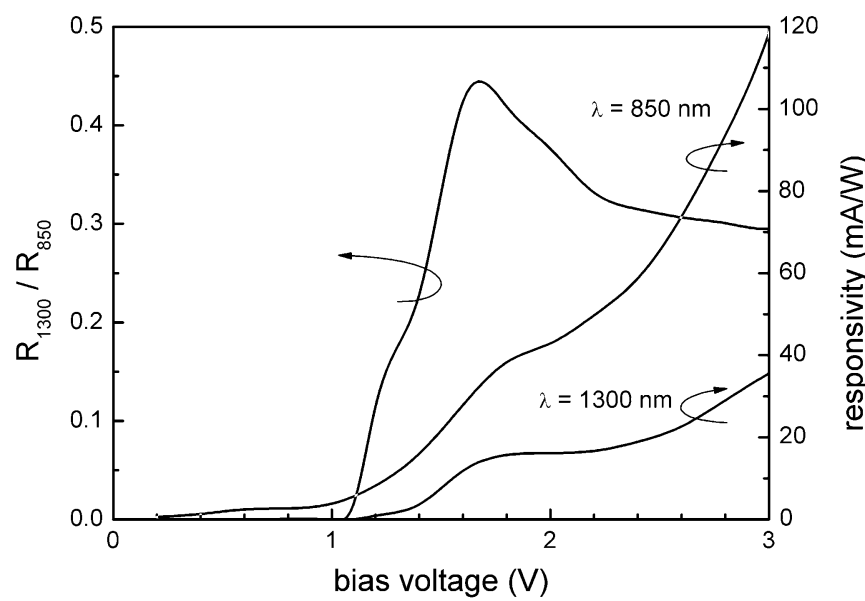

Fig. 5. Voltage-dependence of the responsivity at 850 and $1300 \mathrm{~nm}$ (right scale) and of the responsivity ratio (left scale).

in a reduction of the reponsivity ratio. This behavior is due to the penetration of the depletion region into the InAlAs buffer layer.

\section{CONCLUSION}

We present a planar photodetector that requires only HEMT layer structure and fabrication technology. The spectral responsivity is tunable by the bias voltage making the device applicable as two-color photodetector. A detector fabricated in the InAlAs-InGaAs material system detects and discriminates light originated by GaAs (800-860 nm) and by InP (1300, $1550 \mathrm{~nm}$ ) based optoelectronic technology. The responsivity of the presented device can be improved by increasing the thickness of the InGaAs absorption layer and by using transparent Schottky electrodes [12]. In fact, MSM-2DEG diodes that were optimized as high frequency photodetectors at $1300-\mathrm{nm}$ wavelength have shown responsivities up to $0.61 \mathrm{~A} / \mathrm{W}$ [13]. An alternative method to increase the responsivity is to design the device as edge-coupled photodetector [14]. The extension of the device structure by a third layer with a further spectral responsivity, also separated by a conductive channel, allows the fabrication of a three-color device.

\section{ACKNOWLEDGMENT}

The authors thank A. Förster for MBE growth of the samples.

\section{REFERENCES}

[1] K. Engelhardt and P. Seitz, "Optimum color filters for CCD digital cameras," Appl. Opt., vol. 32, pp. 3015-3023, 1993

[2] D. Knipp, P. G. Herzog, and H. Stiebig, "Stacked amorphous silicon color sensors," IEEE Trans. Electron Devices, vol. 49, pp. 170-176, Jan. 2002.

[3] P. Gwynne, "Tricolor sensors create a sharper image," IEEE Spectr, vol. 39, pp. 23-24, May 2002.

[4] A. Majumdar, K. K. Choi, J. L. Reno, L. P. Rokhinson, and D. C. Tsui, "Two-color quantum-well infrared photodetector with large tunable peaks," Appl. Phys. Lett., vol. 80, pp. 707-709, 2002.

[5] M. Marso, M. Horstmann, H. Hardtdegen, P. Kordoš, and H. Lüth, "Electrical behavior of the InP/InGaAs based MSM-2DEG diode," Solid-State Electron., vol. 41, pp. 25-31, 1997.

[6] M. Marso, M. Horstmann, K. Schimpf, J. Muttersbach, H. Hardtdegen, G. Jacob, and P. Kordoš, "High bandwidth InP/InGaAs based MSM-2DEG diodes for optoelectronic application," in Proc. 9th Int. Conf. Indium Phosphide and Related Materials, Hyannis, MA, 1997, pp. $494-497$.

[7] M. Marso, M. Wolter, P. Javorka, A. Fox, and P. Kordoš, "AlGaN/GaN varactor diode for integration in HEMT circuits," Electron. Lett., vol. 37, pp. 1476-1478, 2001.

[8] M. Marso, J. Bernát, M. Wolter, P. Javorka, A. Fox, and P. Kordoš, "MSM diodes based on an AlGaN/GaN HEMT layer structure for varactor and photodiode application," in Proc. 4th Int. Conf. Advanced Semiconductor Devices and Microsystems, 2002, ISBN 0-7803-7276-X, pp. 295-298.

[9] U. Hodel, A. Orzati, M. Marso, O. Homan, A. Fox, A. v.d. Hart, A Förster, P. Kordoš, and H. Lüth, "A novel InAlAs/InGaAs layer structure for monolithically integrated photoreceiver," in Proc. 2000 Int. Conf. Indium Phosphide and Related Materials, Williamsburg, VA, 2000, pp. 466-469.

[10] M. Marso, P. Gersdorf, A. Fox, A. Förster, U. Hodel, R. Lambertini, and P. Kordoš, "An InAlAs-InGaAs OPFET with responsivity above 200 A/W at $1.3 \mu \mathrm{m}$ wavelength," IEEE Photon. Technol. Lett., vol. 11, pp. 117-119, Jan. 1999.

[11] S. Nojima and K. Watika, "Optimization of quantum well materials and structures for excitonic electroabsorption effects," Appl. Phys. Lett, vol. 53, pp. 1958-1960, 1988.

[12] W. A. Wohlmuth, J.-W. Seo, P. Fay, C. Caneau, and I. Adesida, "A high-speed ITO-InAlAs-InGaAs Schottky-barrier photodetector," IEEE Photon. Technol. Lett., vol. 9, pp. 1388-1390, Oct. 1997.

[13] M. Horstmann, M. Marso, J. Muttersbach, K. Schimpf, and P. Kordoš, "Responsivity enhancement of InGaAs based MSM photodetectors using 2DEG layer sequence and semitransparent electrodes," Electron. Lett., vol. 32, pp. 1613-1614, 1996.

[14] K. Kato, "Ultrawide-band/high-frequency photodetectors," IEEE Trans. Microwave Theory Tech., vol. 47, pp. 1265-1281, July 1999. 